\title{
NEGATIVE CORRELATION IN GRAPHS AND MATROIDS
}

\author{
CHARLES SEMPLE AND DOMINIC WELSH
}

\begin{abstract}
The following two conjectures arose in the work of Grimmett and Winkler, and Pemantle: the uniformly random forest $F$ and the uniformly random connected subgraph $C$ of a finite graph $G$ have the edge-negative-association property. In other words, for all distinct edges $e$ and $f$ of $G$, the probability that $F$ (respectively, $C$ ) contains $e$ conditioned on containing $f$ is less than or equal to the probability that $F$ (respectively, $C$ ) contains $e$. Grimmett and Winkler showed that the first conjecture is true for all simple graphs on 8 vertices and all graphs on 9 vertices with at most 18 edges. In this paper, we describe an infinite, nontrivial class of graphs and matroids for which a generalized version of both conjectures holds.
\end{abstract}

\section{INTRODUCTION}

It follows from the work of Kirchhoff (1847) that the spanning trees of a connected graph satisfy the following negative correlation inequality: if $T$ is a spanning tree of $G$ chosen uniformly at random, then

$$
\mathbb{P}(T \text { contains } e \mid T \text { contains } f) \leq \mathbb{P}(T \text { contains } e)
$$

for all distinct edges $e$ and $f$ of $G$. In other words, the event ' $T$ contains $e$ ' and the event ' $T$ contains $f$ ' are negatively correlated.

The classical FKG inequality [6] stimulated the interest in correlation inequalities in combinatorics. In 1975, Seymour and Welsh [11] considered which matroids have the property that if $B$ is a base of a matroid $M$ chosen uniformly at random, and $e$ and $f$ are two distinct elements of $E(M)$, then

$$
\mathbb{P}(B \text { contains } e \mid B \text { contains } f) \leq \mathbb{P}(B \text { contains } e) .
$$

Such matroids are now called negatively correlated. It was shown in [11] that a particular 8-element binary matroid (now known as $S_{8}$, see [8]) is not negatively correlated. Feder and Mihail [5] enhanced interest in negative correlation in matroids by introducing the class of balanced matroids. A

Date: 30 November 2005.

1991 Mathematics Subject Classification. 05B35.

Key words and phrases. Negatively correlated, balanced matroids, Rayleigh matroids, random cluster model.

The first author was supported by a University of Canterbury Research Grant. This work was done while the second author was an Oxford Fellow at the University of Canterbury. 
matroid is balanced if it and all its minors are negatively correlated, and it is shown in [5] that for balanced matroids the unbiased random walk on the bases-exchange graph is rapidly mixing (for more details, see [7]).

More recently, Grimmett and Winkler [4], and Pemantle [10] have independently made the following two counting conjectures about graphs. For a finite graph $G$ and subset $A$ of edges of $G$, let $F_{A}(G)$ denote the number of forests of $G$ containing $A$ and let $C_{A}(G)$ denote the number of connected subgraphs of $G$ containing $A$.

Conjecture 1.1. For any finite graph $G$ and distinct edges $e$ and $f$ of $G$,

$$
F_{\{e\}}(G) F_{\{f\}}(G)-F_{\{e, f\}}(G) F(G) \geq 0 .
$$

Conjecture 1.2. For any finite graph $G$ and distinct edges $e$ and $f$ of $G$,

$$
C_{\{e\}}(G) C_{\{f\}}(G)-C_{\{e, f\}}(G) C(G) \geq 0 .
$$

In the terminology of [4], $G$ satisfies the inequalities in Conjectures 1.1 and 1.2, respectively, if and only if the uniform forest and the uniform connected subgraph have the edge-negative-association property.

We remark here that, while Conjectures 1.1 and 1.2 are stated in terms of subsets of edges of a graph $G$ that contain certain edges of $G$, these conjectures can be restated in terms of subsets of edges of $G$ that avoid certain edges. In particular, if $F^{A}(G)$ and $C^{A}(G)$ denote the number of forests of $G$ not containing any elements in $A$ and the number of connected subgraphs of $G$ not containing any elements in $A$, respectively, then it is easily checked that Conjectures 1.1 and 1.2 are equivalent to

$$
F^{\{e\}}(G) F^{\{f\}}(G)-F^{\{e, f\}}(G) F(G) \geq 0
$$

and

$$
C^{\{e\}}(G) C^{\{f\}}(G)-C^{\{e, f\}}(G) C(G) \geq 0,
$$

respectively.

Conjectures 1.1 and 1.2 are special cases of more general questions arising from the random-cluster model of statistical mechanics but positive evidence for their truth is limited. By direct numerical computation, Grimmett and Winkler [4] verified Conjecture 1.1 for all simple graphs with at most 8 vertices, and all graphs with 9 vertices and at most 18 edges.

One consequence of the results in this paper is that we can extend the class of graphs for which the conjectures are true to include the infinite class consisting of all graphs that can be obtained by starting with a graph whose maximal 2-connected subgraphs are $K_{4}$ or one of its minors, and repeatedly adding edges in series and in parallel. To do this, we consider a more general question on matroids where the elements are positively weighted. This 
approach was suggested by Alan Sokal (see [4]) and is a technique which has been extremely fruitful over the last few years, see for example $[1,12]$ and the references therein, particularly [9].

The paper is organized as follows. In the next section, we introduce the classes of independence and spanning correlated matroids. In Section 3, we establish a number of basic properties of these classes. Section 4 contains some applications of the results in Section 3 including the proof of the result described in the last paragraph. In the last section, we describe some open questions. Throughout the paper, notation and terminology follows Oxley [8]. For brevity, we will write any 1-element set, $\{x\}$ say, or 2-element set, $\{x, y\}$ say, that appears as a superscript or a subscript as $x$ and $x y$, respectively.

Lastly, we apologize for the somewhat ambiguous use of the word 'correlated' in this paper in connection with what are really negatively correlated events.

\section{Independence and Spanning Correlated Matroids}

Let $M$ be a matroid with ground set $E$. Let $\mathbf{y}: E \rightarrow \mathbb{R}^{+}$be a positive realvalued weighting of $E$. We sometimes write $\mathbf{y}>0$ to denote that $\mathbf{y}(x)>0$ for all $x \in E$. For a subset $A$ of $E$, set $\mathbf{y}(A)=1$ if $A$ is the empty set and set $\mathbf{y}(A)=\prod_{a \in A} \mathbf{y}(a)$ if $A$ is not the empty set. For disjoint subsets $U$ and $V$ of $E$, set

$$
B_{U}^{V}(M ; \mathbf{y})=\sum \mathbf{y}(A)
$$

where the summation is over all bases of $M$ that contain $U$ and avoid $V$, that is, contain all elements in $U$ but no elements in $V$.

Motivated by a classical theorem of Rayleigh about electrical networks. Choe and Wagner [2] introduced the class of Rayleigh matroids. A matroid $M$ with ground set $E$ is Rayleigh if, for all distinct $e, f \in E$ and positive real-valued weightings $\mathbf{y}$ of $E$,

$$
\Delta_{B}(M ; \mathbf{y})=B_{e}(M ; \mathbf{y}) B_{f}(M ; \mathbf{y})-B_{e f}(M ; \mathbf{y}) B(M ; \mathbf{y}) \geq 0 .
$$

Analogously, we define the classes of independence and spanning correlated matroids. For disjoint subsets $U$ and $V$ of $E$, set

$$
I_{U}^{V}(M ; \mathbf{y})=\sum \mathbf{y}(A)
$$

and

$$
S_{U}^{V}(M ; \mathbf{y})=\sum \mathbf{y}(A)
$$

where the summations are over all independent sets and spanning sets of $M$, respectively, that contain $U$ and avoid $V$. Note that each of $B_{U}^{V}(M ; \mathbf{y})$, $I_{U}^{V}(M ; \mathbf{y})$, and $S_{U}^{V}(M ; \mathbf{y})$ can be viewed as an evaluation of a multivariate polynomial in which the variables are the indeterminates $\mathbf{y}(e)$ for all $e \in E$ and the degree of any variable is at most 1 . A matroid $M$ is independence 
correlated if, for all distinct $e, f \in E$ and positive real-valued weightings $\mathbf{y}$ of $E$,

$$
\Delta_{I}(M ; \mathbf{y})=I_{e}(M ; \mathbf{y}) I_{f}(M ; \mathbf{y})-I_{e f}(M ; \mathbf{y}) I(M ; \mathbf{y}) \geq 0 .
$$

A matroid is spanning correlated if, for all distinct $e, f \in E$ and positive real-valued weightings $\mathbf{y}$ of $E$,

$$
\Delta_{S}(M ; \mathbf{y})=S_{e}(M ; \mathbf{y}) S_{f}(M ; \mathbf{y})-S_{e f}(M ; \mathbf{y}) S(M ; \mathbf{y}) \geq 0 .
$$

We say a matroid is correlated if it is both independence correlated and spanning correlated. Strictly speaking, each of $\Delta_{B}(M ; \mathbf{y}), \Delta_{I}(M ; \mathbf{y})$, and $\Delta_{S}(M ; \mathbf{y})$ depend on $e$ and $f$. However, for ease of reading, this is omitted in the ' $\Delta$ ' notation.

An immediate consequence of the definition of independence correlated is that if $G$ is a graph with edge set $E$ and $M(G)$ is independence correlated, then, by setting the weighting $\mathbf{y}$ on $E$ to be $\mathbf{y}(x)=1$ for all $x \in E$, we have that $G$ satisfies Conjecture 1.1. An analogous consequence follows from the definition of spanning correlated. In particular, we have the following proposition.

\section{Proposition 2.1.}

(i) If the class of graphic matroids is independence correlated, then Conjecture 1.1 holds for all graphs.

(ii) If the class of graphic matroids is spanning correlated, then Conjecture 1.2 holds for all graphs.

Trivially, all matroids of rank at most 1 are independence correlated and it is easily seen that all rank- 2 matroids are independence correlated. The next example shows that all uniform matroids are independence correlated.

Example 2.2. Uniform matroids are correlated. Let $\left\{e_{1}, e_{2}, \ldots, e_{n}\right\}$ denote the ground set of the uniform matroid $U_{r, n}$, where $r \geq 2$. Let $\mathbf{y}$ be a positive real-valued weighting of the ground set, where $y_{i}=\mathbf{y}\left(e_{i}\right)$. Choosing two distinct elements of the ground set, we may assume without loss of generality that these elements are $e_{n-1}$ and $e_{n}$. For all $k \geq 0$, let $s_{k}$ denote the $k$-th elementary symmetric function

$$
\sum y_{i_{1}} y_{i_{2}} \cdots y_{i_{k}}
$$

where the summation is over all distinct $k$ element subsets $\left\{i_{1}, i_{2}, \ldots, i_{k}\right\}$ of $\{1,2, \ldots, n-2\}$. We show in Lemma 3.1 that, for distinct elements $e$ and $f$ of a matroid $M$,

$I_{e}(M ; \mathbf{y}) I_{f}(M ; \mathbf{y})-I_{e f}(M ; \mathbf{y}) I(M ; \mathbf{y})=I_{e}^{f}(M ; \mathbf{y}) I_{f}^{e}(M ; \mathbf{y})-I_{e f}(M ; \mathbf{y}) I^{e f}(M ; \mathbf{y})$.

Therefore $U_{r, n}$ is independence correlated if and only if

$$
y_{n-1}\left(\sum_{k=0}^{r-1} s_{k}\right) y_{n}\left(\sum_{k=0}^{r-1} s_{k}\right)-y_{n-1} y_{n}\left(\sum_{k=0}^{r-2} s_{k}\right)\left(\sum_{k=0}^{r} s_{k}\right) \geq 0 .
$$


In particular, $U_{r, n}$ is independence correlated if and only if

$$
\left(\sum_{k=0}^{r-1} s_{k}\right)^{2} \geq\left(\sum_{k=0}^{r-2} s_{k}\right)\left(\sum_{k=0}^{r} s_{k}\right) .
$$

Writing $S_{k}=\sum_{i=0}^{k} s_{i}$, this reduces to

$$
\left(S_{r-2}+s_{r-1}\right)^{2} \geq S_{r-2}\left(S_{r-2}+s_{r-1}+s_{r}\right)
$$

or, equivalently,

$$
s_{r-1}^{2}+s_{r-1} S_{r-2}-s_{r} S_{r-2} \geq 0 .
$$

Elementary algebra shows that this last inequality does indeed hold since the left-hand-side is a multivariate polynomial with nonnegative coefficients.

It will follow from further results later in the paper that all uniform matroids are also spanning correlated, and so the class of uniform matroids is contained in the class of correlated matroids.

\section{Properties of Independence and Spanning Correlated MATROIDS}

In this section, we establish several attractive properties of independence and spanning correlated matroids. We begin with some simple, but useful, identities.

Lemma 3.1. Let $M$ be a matroid with ground set $E$, let $e$ and $f$ be distinct elements of $E$, and let $\mathbf{y}$ be a positive real-valued weighting of $E$. Then the following quantities are equal:

(i) $I_{e}(M ; \mathbf{y}) I_{f}(M ; \mathbf{y})-I_{e f}(M ; \mathbf{y}) I(M ; \mathbf{y})$;

(ii) $I^{e}(M ; \mathbf{y}) I^{f}(M ; \mathbf{y})-I^{e f}(M ; \mathbf{y}) I(M ; \mathbf{y})$;

(iii) $I_{e}^{f}(M ; \mathbf{y}) I_{f}^{e}(M ; \mathbf{y})-I_{e f}(M ; \mathbf{y}) I^{e f}(M ; \mathbf{y})$; and

(iv) $I^{e}(M ; \mathbf{y}) I_{e}^{f}(M ; \mathbf{y})-I_{e}(M ; \mathbf{y}) I^{e f}(M ; \mathbf{y})$.

Furthermore, the analogous quantities are equal for the spanning sets of $M$.

Proof. We first show that (i) and (iii) are equal. For distinct elements $e, f \in$ $E$ and a positive real-valued weighting $\mathbf{y}$ of $E$,

$$
\begin{aligned}
I_{e}(M ; \mathbf{y}) I_{f}(M ; \mathbf{y})-I_{e f}(M ; \mathbf{y}) I(M ; \mathbf{y}) & \\
= & \left(I_{e f}(M ; \mathbf{y})+I_{e}^{f}(M ; \mathbf{y})\right)\left(I_{e f}(M ; \mathbf{y})+I_{f}^{e}(M ; \mathbf{y})\right) \\
& \quad-I_{e f}(M ; \mathbf{y})\left(I_{e f}(M ; \mathbf{y})+I_{e}^{f}(M ; \mathbf{y})+I_{f}^{e}(M ; \mathbf{y})+I^{e f}(M ; \mathbf{y})\right) \\
= & I_{e}^{f}(M ; \mathbf{y}) I_{f}^{e}(M ; \mathbf{y})-I_{e f}(M ; \mathbf{y}) I^{e f}(M ; \mathbf{y}) .
\end{aligned}
$$

A similar argument shows that (ii) and (iii) are equal. To see that (iv) is equal to (iii), replace $I^{e}(M ; \mathbf{y})$ and $I_{e}(M ; \mathbf{y})$ in (iv) with $I_{f}^{e}(M ; \mathbf{y})+$ 
$I^{e f}(M ; \mathbf{y})$ and $I_{e f}(M ; \mathbf{y})+I_{e}^{f}(M ; \mathbf{y})$, respectively, and simplify. The proof for spanning sets is similar and omitted.

The proof of the next result is straightforward and omitted.

Proposition 3.2. The classes of independence correlated and spanning correlated matroids are each closed under direct sums.

The proofs of Propositions 3.3 and 3.5 use techniques used extensively in [1] and [2], but for the sake of completeness we include full details here.

Proposition 3.3. A matroid $M$ is independence correlated if and only if $M^{*}$ is spanning correlated.

Proof. Let $M$ be a matroid with ground set $E$, and suppose that $M$ is independence correlated. Let $\mathbf{y}$ be a positive real-valued weighting of $E$ and let $\mathbf{y}^{-1}$ denote the positive real-valued weighting of $E$ obtained from $\mathbf{y}$ by setting $\mathbf{y}^{-1}(x)=\frac{1}{\mathbf{y}(x)}$ for all $x \in E$. If $U$ and $V$ are disjoint subsets of $E$, then, as $A$ is independent in $M$ if and only if $E-A$ is spanning in $M^{*}$,

$$
S_{U}^{V}\left(M^{*} ; \mathbf{y}\right)=\mathbf{y}^{E} I_{V}^{U}\left(M ; \mathbf{y}^{-1}\right) .
$$

Since $M$ is independence correlated, it follows that, for all distinct $e, f \in E$,

$$
S^{e}(M ; \mathbf{y}) S^{f}(M ; \mathbf{y})-S^{e f}(M ; \mathbf{y}) S(M ; \mathbf{y}) \geq 0,
$$

which, by Lemma 3.1, implies that

$$
\Delta_{S}(M ; \mathbf{y})=S_{e}(M ; \mathbf{y}) S_{f}(M ; \mathbf{y})-S_{e f}(M ; \mathbf{y}) S(M ; \mathbf{y}) \geq 0 .
$$

Thus $M^{*}$ is spanning correlated. The proof of the converse is similar and omitted.

The following result is an immediate consequence of Proposition 3.3.

Corollary 3.4. The class of correlated matroids is closed under duality.

Whether the class of independence correlated matroids, and thus the class of spanning correlated matroids, is closed under duality is unclear. However, both classes are closed under minors.

Proposition 3.5. The classes of independence correlated matroids and spanning correlated matroids are each closed under minors.

Proof. Suppose that $M$ is an independence correlated matroid with ground set $E$ and let $a \in E$. Let $\mathbf{y}_{a}$ be a positive real-valued weighting of $E-\{a\}$. Let $\mathbf{y}$ be the weighting of $E$ that is obtained by setting $\mathbf{y}(x)=\mathbf{y}_{a}(x)$ for all 


$$
\begin{aligned}
& x \in E-\{a\} \text { and } \mathbf{y}(a)=\epsilon>0 \text {. Now } \\
& \Delta_{I}(M ; \mathbf{y})=I_{e}(M ; \mathbf{y}) I_{f}(M ; \mathbf{y})-I_{e f}(M ; \mathbf{y}) I(M ; \mathbf{y}) \\
& =\left(I_{e}^{a}(M ; \mathbf{y})+I_{e a}(M ; \mathbf{y})\right)\left(I_{f}^{a}(M ; \mathbf{y})+I_{f a}(M ; \mathbf{y})\right) \\
& -\left(I_{e f}^{a}(M ; \mathbf{y})+I_{e f a}(M ; \mathbf{y})\right)\left(I^{a}(M ; \mathbf{y})+I_{a}(M ; \mathbf{y})\right) \\
& =\left(I_{e}^{a}(M ; \mathbf{y}) I_{f}^{a}(M ; \mathbf{y})-I_{e f}^{a}(M ; \mathbf{y}) I^{a}(M ; \mathbf{y})\right)+\epsilon P\left(\mathbf{y}_{a}\right) \\
& +\left(I_{e a}(M ; \mathbf{y}) I_{f a}(M ; \mathbf{y})-I_{e f a}(M ; \mathbf{y}) I_{a}(M ; \mathbf{y})\right) \text {, }
\end{aligned}
$$

where $P\left(\mathbf{y}_{a}\right)$ is a polynomial in $\mathbf{y}_{a}$. Since

$$
\Delta_{I}\left(M \backslash a ; \mathbf{y}_{a}\right)=I_{e}^{a}(M ; \mathbf{y}) I_{f}^{a}(M ; \mathbf{y})-I_{e f}^{a}(M ; \mathbf{y}) I^{a}(M ; \mathbf{y})
$$

and

$$
\epsilon^{2} \Delta_{I}\left(M / a ; \mathbf{y}_{a}\right)=I_{e a}(M ; \mathbf{y}) I_{f a}(M ; \mathbf{y})-I_{e f a}(M ; \mathbf{y}) I_{a}(M ; \mathbf{y}),
$$

we deduce that

$$
\Delta_{I}(M ; \mathbf{y})=\Delta_{I}\left(M \backslash a ; \mathbf{y}_{a}\right)+\epsilon P\left(\mathbf{y}_{a}\right)+\epsilon^{2} \Delta_{I}\left(M / a ; \mathbf{y}_{a}\right) .
$$

We first show that $M \backslash a$ is independence correlated. Since $M$ is independence correlated,

$$
\lim _{\epsilon \rightarrow 0^{+}} \Delta_{I}(M ; \mathbf{y})=\Delta_{I}\left(M \backslash a ; \mathbf{y}_{a}\right) \geq 0 .
$$

It follows that $M \backslash a$ is independence correlated. To see that $M / a$ is independence correlated, multiply both sides of (1) by $\epsilon^{-2}>0$ to get

$$
\Delta_{I}\left(M / a ; \mathbf{y}_{a}\right)=\lim _{\epsilon \rightarrow \infty} \frac{1}{\epsilon^{2}} \Delta_{I}(M ; \mathbf{y}) \geq 0
$$

as $M$ is independence correlated. Thus the class of independence correlated matroids is closed under minors. The fact that the class of spanning correlated matroids is closed under minors is a consequence of this and Proposition 3.3.

Despite Proposition 3.5, we do not know whether or not the class of graphs which satisfy Conjecture 1.1 is closed under minors. However, if $G$ is a graph and we know that $M(G)$ is independence correlated, then it immediately follows from Proposition 3.5 that all minors of $G$ satisfy Conjecture 1.1.

Corollary 3.6 is an immediate consequence of Proposition 3.5.

Corollary 3.6. The class of correlated matroids is closed under minors.

Let $M$ be a matroid with ground set $E$, let $a \in E$, and let $b$ and $c$ be distinct elements not in $E$. Let $N$ be the matroid obtained from $M$ by replacing $a$ with $b$ and $c$ in parallel so that both $N \backslash b$ and $N \backslash c$ are isomorphic to $M$. We say that $N$ is a parallel extension of $M$. Now let $N$ be the matroid obtained from $M$ by replacing $a$ with $b$ and $c$ in series so that both $N / b$ and $N / c$ are isomorphic to $M$. In this case, we say that $N$ is a series extension of $M$. For graphs, a series extension of $G$ corresponds to replacing an edge by a 2-edge path. 
Proposition 3.7. Let $M$ be a matroid. Then the following hold.

(i) If $M$ is independence correlated, then every series-parallel extension of $M$ is independence correlated.

(ii) If $M$ is spanning correlated, then every series-parallel extension of $M$ is spanning correlated.

Before proving Proposition 3.7 we emphasize again that we are unable to show that if a graph $G$ satisfies Conjecture 1.1 (or Conjecture 1.2), then so does any series or parallel extension of $G$.

Proof of Proposition 3.7. Since a matroid $N$ is a parallel extension of a matroid $M$ if and only if $N^{*}$ is a series extension of $M^{*}$, it suffices to show by Proposition 3.3 that (i) holds.

Let $M$ be independence correlated and suppose first that $N$ is a parallel extension of $M$ with $b$ and $c$ replacing $a$. Let $\mathbf{y}_{N}$ be a positive real-valued weighting of $E(N)$. Let $\mathbf{y}_{M}$ be the weighting on $E(M)$ that is obtained from $\mathbf{y}_{N}$ by setting $\mathbf{y}_{M}(x)=\mathbf{y}_{N}(x)$ for all $x \in E(M)-\{a\}$ and $\mathbf{y}_{M}(a)=1$. If $U$ and $V$ are disjoint subsets of $E(N)-\{b, c\}$, then

$$
\begin{aligned}
I_{U}^{V}\left(N ; \mathbf{y}_{N}\right) & =I_{U \cup\{b\}}^{V}\left(N ; \mathbf{y}_{N}\right)+I_{U \cup\{c\}}^{V}\left(N ; \mathbf{y}_{N}\right)+I_{U}^{V \cup\{b, c\}}\left(N ; \mathbf{y}_{N}\right) \\
& =\mathbf{y}_{N}(b) I_{U \cup\{a\}}^{V}\left(M ; \mathbf{y}_{M}\right)+\mathbf{y}_{N}(c) I_{U \cup\{a\}}^{V}\left(M ; \mathbf{y}_{M}\right)+I_{U}^{V \cup\{a\}}\left(M ; \mathbf{y}_{M}\right) \\
& =\left(\mathbf{y}_{N}(b)+\mathbf{y}_{N}(c)\right) I_{U \cup\{a\}}^{V}\left(M ; \mathbf{y}_{M}\right)+I_{U}^{V \cup\{a\}}\left(M ; \mathbf{y}_{M}\right) \\
& =I_{U \cup\{a\}}^{V}\left(M ; \mathbf{y}_{M}^{\prime}\right)+I_{U}^{V \cup\{a\}}\left(M ; \mathbf{y}_{M}^{\prime}\right)=I_{U}^{V}\left(M ; \mathbf{y}_{M}^{\prime}\right),
\end{aligned}
$$

where $\mathbf{y}_{M}^{\prime}$ is obtained from $\mathbf{y}_{M}$ by setting $\mathbf{y}_{M}^{\prime}(x)=\mathbf{y}_{M}(x)$ for all $x \in$ $E(M)-\{a\}$ and $\mathbf{y}_{M}^{\prime}(a)=\mathbf{y}_{N}(b)+\mathbf{y}_{N}(c)$. Since $\mathbf{y}_{M}^{\prime}>0$ and $M$ is independence correlated, $\Delta_{I}\left(M ; \mathbf{y}_{M}^{\prime}\right) \geq 0$. It now follows that $\Delta_{I}\left(N ; \mathbf{y}_{N}\right) \geq 0$ for all distinct elements $e, f \in E(N)-\{b, c\}$.

Now assume that $\{b, c\} \cap\{e, f\}$ is nonempty. If $\{b, c\}=\{e, f\}$, then $I_{e f}\left(N ; \mathbf{y}_{N}\right)=0$, so in this case $\Delta_{I}\left(N ; \mathbf{y}_{N}\right) \geq 0$. If $\{b, c\} \cap\{e, f\}$ meets in exactly one element, $\{b\}$ say, then, using the weighting $\mathbf{y}_{M}^{\prime}$ above in which $\mathbf{y}_{M}^{\prime}(a)=\mathbf{y}_{N}(b)+\mathbf{y}_{N}(c)$, we get

$$
\Delta_{I}\left(N ; \mathbf{y}_{N}\right)=\frac{\mathbf{y}_{N}(b)}{\mathbf{y}_{N}(b)+\mathbf{y}_{N}(c)} \Delta_{I}\left(M ; \mathbf{y}_{M}^{\prime}\right) \geq 0 .
$$

It now follows that $N$ is independence correlated.

Now suppose that $N$ is a series extension of $M$ with $b$ and $c$ replacing $a$. Let $\mathbf{y}_{N}$ be a positive real-valued weighting of $E(N)$. Let $\mathbf{y}_{M}$ be the weighting on $E(M)$ that is obtained from $\mathbf{y}_{N}$ by setting $\mathbf{y}_{M}(x)=\mathbf{y}_{N}(x)$ for all $x \in E(M)-\{a\}$ and $\mathbf{y}_{M}(a)=1$. If $U$ and $V$ are disjoint subsets of $E(N)-\{b, c\}$, then, by similar arguments to that of the parallel case,

$$
\frac{1}{\mathbf{y}_{N}(b)+\mathbf{y}_{N}(c)+1} I_{U}^{V}\left(N ; \mathbf{y}_{N}\right)=I_{U}^{V}\left(M ; \mathbf{y}_{M}^{\prime}\right),
$$


where $\mathbf{y}_{M}^{\prime}$ is obtained from $\mathbf{y}_{M}$ by setting $\mathbf{y}_{M}^{\prime}(x)=\mathbf{y}_{M}(x)$ for all $x \in$ $E(M)-\{a\}$ and

$$
\mathbf{y}_{M}^{\prime}(a)=\frac{\mathbf{y}_{N}(b) \mathbf{y}_{N}(c)}{\mathbf{y}_{N}(b)+\mathbf{y}_{N}(c)+1} .
$$

Since $M$ is independence correlated and $\mathbf{y}_{M}^{\prime}>0$, we deduce that $\Delta_{I}\left(N ; \mathbf{y}_{N}\right) \geq 0$ for all distinct $e, f \in E(N)-\{b, c\}$.

Assume that $\{e, f\}=\{b, c\}$. By Lemma 3.1,

$$
\begin{aligned}
\Delta_{I}\left(N ; \mathbf{y}_{N}\right)= & I_{b}^{c}\left(N ; \mathbf{y}_{N}\right) I_{c}^{b}\left(N ; \mathbf{y}_{N}\right)-I_{b c}\left(N ; \mathbf{y}_{N}\right) I^{b c}\left(N ; \mathbf{y}_{N}\right) \\
= & \mathbf{y}_{N}(b) I^{a}\left(M ; \mathbf{y}_{M}\right) \mathbf{y}_{N}(c) I^{a}\left(M ; \mathbf{y}_{M}\right) \\
& -\mathbf{y}_{N}(b) \mathbf{y}_{N}(c) I_{a}\left(M ; \mathbf{y}_{M}\right) I^{a}\left(M ; \mathbf{y}_{M}\right) \\
= & \mathbf{y}_{N}(b) \mathbf{y}_{N}(c) I^{a}\left(M ; \mathbf{y}_{M}\right)\left(I^{a}\left(M ; \mathbf{y}_{M}\right)-I_{a}\left(M ; \mathbf{y}_{M}\right)\right) .
\end{aligned}
$$

Thus, as $\mathbf{y}_{M}(a)=1$, we have $\Delta_{I}\left(N ; \mathbf{y}_{N}\right) \geq 0$ if and only if

$$
I^{a}\left(M ; \mathbf{y}_{M}\right)-I_{a}\left(M ; \mathbf{y}_{M}\right)=I^{a}\left(M ; \mathbf{y}_{M}\right)-I\left(M / a ; \mathbf{y}_{M}\right) \geq 0 .
$$

Since every independent set of $M / a$ is an independent set of $M$ that avoids $a$, it follows that $\Delta_{I}\left(N ; \mathbf{y}_{N}\right) \geq 0$.

Lastly, assume that $\{b, c\}$ and $\{e, f\}$ meet in exactly one element, $b=e$ say. Then, by Lemma 3.1,

$$
\begin{aligned}
\Delta_{I}\left(N ; \mathbf{y}_{N}\right) & =I_{b}^{f}\left(N ; \mathbf{y}_{N}\right) I_{f}^{b}\left(N ; \mathbf{y}_{N}\right)-I_{b f}\left(N ; \mathbf{y}_{N}\right) I^{b f}\left(N ; \mathbf{y}_{N}\right) \\
& =\mathbf{y}_{N}(b) I^{f}\left(N / b ; \mathbf{y}_{N}\right) I_{f}\left(N \backslash b ; \mathbf{y}_{N}\right)-\mathbf{y}_{N}(b) I_{f}\left(N / b ; \mathbf{y}_{N}\right) I^{f}\left(N \backslash b ; \mathbf{y}_{N}\right) .
\end{aligned}
$$

Let $\mathbf{y}_{M}^{\prime \prime}$ be the positive real-valued weighting on $E(M)$ that is obtained by setting $\mathbf{y}_{M}^{\prime \prime}(x)=\mathbf{y}_{N}(x)$ for all $x \in E(M)-\{a\}$ and $\mathbf{y}_{M}^{\prime \prime}(a)=\mathbf{y}_{N}(c)$. Then, as

and

$$
I_{f}\left(N \backslash b ; \mathbf{y}_{N}\right)=I_{f}^{a}\left(M ; \mathbf{y}_{M}^{\prime \prime}\right)+\mathbf{y}_{N}(c) I_{f}^{a}\left(M ; \mathbf{y}_{M}^{\prime \prime}\right)
$$

$$
I^{f}\left(N \backslash b ; \mathbf{y}_{N}\right)=I^{f a}\left(M ; \mathbf{y}_{M}^{\prime \prime}\right)+\mathbf{y}_{N}(c) I^{f a}\left(M ; \mathbf{y}_{M}^{\prime \prime}\right),
$$

we see that $\Delta_{I}\left(N ; \mathbf{y}_{N}\right) \geq 0$ holds if and only if

$$
\left(1+\mathbf{y}_{N}(c)\right)\left(I^{f}\left(M ; \mathbf{y}_{M}^{\prime \prime}\right) I_{f}^{a}\left(M ; \mathbf{y}_{M}^{\prime \prime}\right)-I_{f}\left(M ; \mathbf{y}_{M}^{\prime \prime}\right) I^{f a}\left(M ; \mathbf{y}_{M}^{\prime \prime}\right)\right) \geq 0 .
$$

It now follows by Lemma 3.1 that $\Delta_{I}\left(N ; \mathbf{y}_{N}\right) \geq 0$ whenever $|\{b, c\} \cap\{e, f\}|=$ 1. Thus $N$ is independence correlated.

Note that the transformation of weights $\mathbf{y}_{N}$ to $\mathbf{y}_{M}^{\prime}$ and $\mathbf{y}_{M}^{\prime \prime}$ used in the proof of Proposition 3.7 are special cases of those used by Sokal to obtain the general formulae (4.22) and (4.28) in [12].

$q$-correlated matroids. Alan Sokal (private communication, 2005) has pointed out a generalization of some of the above to the random cluster model. Call a matroid $M$ with ground set $E$ q-correlated if, for a random subset $R$ of $E$ chosen according to the random cluster measure

$$
\mathbb{P}(A) \propto\left(\prod_{e \in A} p(e) \prod_{e \in E-A}(1-p(e))\right) q^{-r(A)},
$$


we have that

$$
\mathbb{P}(R \text { contains } e) \mathbb{P}(R \text { contains } f) \geq \mathbb{P}(R \text { contains } e \text { and } f)
$$

for all distinct $e, f \in E$ and all probability distributions p. It is easy to see that (2) is equivalent to

$$
\tilde{Z}_{M / e}(q, \mathbf{y}) \tilde{Z}_{M / f}(q, \mathbf{y}) \geq q^{r(\{e\})+r(\{f\})-r(\{e, f\})} \tilde{Z}_{M}(q, \mathbf{y}) \tilde{Z}_{M / e f}(q, \mathbf{y})
$$

for all distinct $e, f \in E$ and $\mathbf{y}>0$. Here $\tilde{Z}$ is the multivariate Tutte polynomial (or almost equivalently the random cluster partition function defined in (1.3) of [12]).

Alternatively, and more generally, we can define the partition function with respect to an arbitrary nonnegative weight function $w$ as follows. Let

$$
\mathbb{P}(A) \propto\left(\prod_{e \in A} p(e) \prod_{e \in E-A}(1-p(e))\right) w(A),
$$

which has an associated partition function

$$
Z(w, \mathbf{y})=\sum_{A \subseteq E}\left(\prod_{e \in A} p(e) \prod_{e \in E-A}(1-p(e))\right) w(A) .
$$

Then following the approach in [12], we see that (2) is equivalent to

$$
\frac{\partial}{\partial y_{e}} Z(w, \mathbf{y}) \frac{\partial}{\partial y_{e}} Z(w, \mathbf{y}) \geq Z(w, \mathbf{y}) \frac{\partial^{2}}{\partial y_{e} \partial y_{f}} Z(w, \mathbf{y})
$$

with $w(A)$ equal to $q^{-r(A)}$ and where $y_{e}=\mathbf{y}(e)$ for all $e$.

Essentially the same arguments as used in this section show that the following statement is true:

- If $M$ is $q$-correlated, then any series-parallel extension of $M$ is $q$ correlated. In particular, series-parallel networks are $q$-correlated for all $0<q \leq 1$.

Furthermore, from (3.10) of [12], we know that with $\mathbf{y}$ fixed

$$
\lim _{q \rightarrow 0} q^{r(E)} \tilde{Z}_{M}(q, \mathbf{y})=S(M ; \mathbf{y})
$$

Similarly, if $q \rightarrow 0$ with $\mathbf{w}=\mathbf{y} / q$ fixed, we get

$$
\lim _{q \rightarrow 0} \tilde{Z}_{M}(q, q \mathbf{w})=I(M ; \mathbf{y})
$$

by (3.12) of [12]. Hence, combining these we get the following statement:

- If $M$ is $q$-correlated for all sufficiently small (but positive) $q$, then $M$ is correlated. 
However, it seems possible (though we have no examples) that there exist matroids which are independence and/or spanning correlated but not $q$ correlated for any $q$, where $0<q<1$.

Note that, for $q \geq 1$, the inequality (2) is reversed for all $e$ and $f$ and all matroids by an easy application of the FKG inequality.

\section{Applications and Examples}

We begin this section by considering a particular subclass of the class of independence correlated matroids. We say that a matroid $M$ is strongly independence correlated if, for all distinct $e$ and $f$ in $E(M)$ and positive real-valued weightings $\mathbf{y}$ of $E$, we have that $\Delta_{I}(M ; \mathbf{y})$ is a multivariate polynomial in which each coefficient is non-negative. As an example, all uniform matroids are strongly correlated. Examination of the proofs of Propositions 3.3, 3.5, and 3.7, show that exactly the same arguments give the following proposition.

Proposition 4.1. Let $M$ be a strongly independence correlated matroid. Then

(i) $M^{*}$ is strongly spanning correlated,

(ii) every minor of $M$ is strongly independence correlated, and

(iii) every series-parallel extension of $M$ is strongly independence correlated.

It can be checked that $M\left(K_{4}\right)$ is not strongly independence correlated, but every proper minor of $M\left(K_{4}\right)$ is strongly independence correlated. Combining this with Proposition 4.1, and the fact that if $M$ is a binary matroid and has no $M\left(K_{4}\right)$-minor, then $M$ is the cycle matroid of a series-parallel network (see [8]), we get the following result.

Theorem 4.2. A binary matroid is strongly independence correlated if and only if it has no $K_{4}$ as a minor.

Computationally, this means that any Maple aided computation to verify that a particular graph or matroid strictly containing $K_{4}$ or $M\left(K_{4}\right)$ as a minor is correlated may need several possibly intractable algebraic manipulations. For example, a Maple calculation to determine whether or not $\Delta_{I}\left(M\left(K_{5}\right) ; \mathbf{y}\right)$ is non-negative for a pair of non-adjacent edges results in having to show that a polynomial of degree 6 consisting of 228 terms in 8 variables is nonnegative for all $\mathbf{y}>0$.

Rayleigh matroids. Comparison of the definitions of Rayleigh matroids and correlated matroids suggests some close connection between the two classes. We show next that the classes of independence and spanning correlated matroids are contained in the class of Rayleigh matroids. 
Proposition 4.3. Let $M$ be a matroid. If $M$ is either independence or spanning correlated, then it is a Rayleigh matroid.

Proof. Let $M$ be an independence correlated matroid with ground set $E$. Suppose that for some choice of $e, f \in E$ and positive real-valued weighting $\mathbf{y}$ of $E$, we have

$$
\Delta_{B}(M ; \mathbf{y})=B_{e}(M ; \mathbf{y}) B_{f}(M ; \mathbf{y})-B_{e f}(M ; \mathbf{y}) B(M ; \mathbf{y})<0 .
$$

Let $\mathbf{y}^{\prime}$ be the positive real-valued weighting that is obtained from $\mathbf{y}$ by multiplying each element by $k>0$. Then

$$
\Delta_{I}\left(M ; \mathbf{y}^{\prime}\right)=I_{e}\left(M ; \mathbf{y}^{\prime}\right) I_{f}\left(M ; \mathbf{y}^{\prime}\right)-I_{e f}\left(M ; \mathbf{y}^{\prime}\right) I\left(M ; \mathbf{y}^{\prime}\right) .
$$

Denoting the rank of $M$ by $r$, it follows that

$$
\begin{aligned}
\Delta_{I}\left(M ; \mathbf{y}^{\prime}\right)=k^{2 r-2}\left(B_{e}(M ; \mathbf{y})\right. & \left.B_{f}(M ; \mathbf{y})-B_{e f}(M ; \mathbf{y}) B(M ; \mathbf{y})\right) \\
& +k^{2 r-3} P_{1}(\mathbf{y})+k^{2 r-4} P_{2}(\mathbf{y})+\cdots+1,
\end{aligned}
$$

where $P_{i}(\mathbf{y})$ is a polynomial in $\mathbf{y}$. Since $M$ is independence correlated and $k$ can be arbitrarily large, this implies that

$$
B_{e}(M ; \mathbf{y}) B_{f}(M ; \mathbf{y})-B_{e f}(M ; \mathbf{y}) B(M ; \mathbf{y}) \geq 0 .
$$

This contradiction to (3) implies that every independence correlated matroid is Rayleigh.

To see that every spanning correlated matroid $N$ is Rayleigh, note that, by Proposition 3.3, $N^{*}$ is independence correlated and so $N^{*}$ is a Rayleigh matroid. Since Rayleigh matroids are closed under duality [2], it follows that $N$ is a Rayleigh matroid.

Choe and Wagner [2] showed that the class of Rayleigh matroids is strictly contained in the class of balanced matroids and this implies that the binary matroid $S_{8}$ is not correlated. (For details of $S_{8}$, see [8].) It is also shown in [2] that the affine geometry $A G(3,2)$ is Rayleigh. Using the very useful properties that $A G(3,2)$ is doubly transitive and self-dual, we were able to show reasonably quickly using Maple that $A G(3,2)$ is correlated. In particular, by considering $\Delta_{I}(A G(3,2) ; \mathbf{y})$ for any pair of elements of $A G(3,2)$, it suffices to show that a degree- 6 multivariate polynomial in 6 variables with 227 terms is non-negative for all $\mathbf{y}$. Fortunately, only 12 of these terms are negative and each such term is of the form $-2 y_{i} y_{j} y_{k} y_{l} y_{m}^{2}$ where $i, j, k, l$, and $m$ are pairwise distinct. The negativity of these terms are easily dealt with by completing the square using the remaining 215 terms. It is worth noting that these are precisely the negative terms that arose in the analogous proof in [2] to show that $A G(3,2)$ is Rayleigh and thus agrees with their computation. Since the class of correlated matroids is closed under minors, this implies that the Fano matroid and its dual as well as $M\left(K_{4}\right)$ are correlated. In particular, combining this with the general results in the last section, we extend the class of graphs satisfying Conjectures 1.1 and 1.2. as follows. 
Theorem 4.4. Let $G$ be a graph that can be obtained by starting with a graph whose maximal 2-connected subgraphs are $K_{4}$ or one of its minors, and repeatedly adding edges in series and in parallel. Then Conjectures 1.1 and 1.2 hold for $G$.

Proof. Since $M\left(K_{4}\right)$ is correlated, $K_{4}$ and its minors are correlated by Proposition 3.5. The theorem now follows by combining Propositions 3.7 and 3.2 .

\section{Some Open Questions}

There are many questions left unanswered. We list some of these in no particular order of importance or difficulty.

(1) Does there exist a Rayleigh matroid that is not correlated?

(2) Choe and Wagner [2] showed that the 2-sum of two Rayleigh matroids is also Rayleigh. However, proving the analogous result for correlated matroids appears more difficult.

(3) Does there exist a graph $G$ that satisfies Conjecture 1.1, but $M(G)$ is not independence correlated?

(4) Is there a matroid that is independence correlated, but is not spanning correlated?

(5) Wagner [13] showed that every rank-3 matroid is Rayleigh. Is every rank-3 matroid correlated?

Note. Since this paper was submitted, two of these open questions have now been settled. Cocks [3] and Wagner [14] have independently proved that the 2-sum of two correlated matroids is correlated, thus answering (2). Furthermore, Cocks [3] has shown that if Conjecture 1.1 holds for a graph $G$, then $M(G)$ is independence correlated, thus settling (3).

\section{ACKNOWLEDGMENTS}

We thank Geoffrey Grimmett and Ben Martin for their help on various points, and Cliff Cocks for pointing out an error in an earlier draft. We thank Mark Hickman for his assistance with Maple computations, and Alan Sokal for originally suggesting (March 2002) that Conjectures 1.1 and 1.2 held for series-parallel networks and for his comments on an earlier draft of this paper.

\section{REFERENCES}

[1] Choe, Y.-B., Oxley, J. G., Sokal, A. D., and Wagner, D. G., Homogenous multivariate polynomials with the half-plane property, Advances in Applied Mathematics 32 (2004), 88-187.

[2] Choe, Y.-B. and Wagner, D. G., Rayleigh matroids, Combinatorics, Probability, and Computing, in press. 
[3] Cocks, C. C., Correlated matroids, submitted.

[4] Grimmett, G. R. and Winkler, S. N., Negative association in uniform forests and connected graphs, Random Structures and Algorithms 24 (2004), 444-460.

[5] Feder, T. and Mihail, M., Balanced matroids, in Proceedings of the 24th ACM Symposium on Theory of Computing, 1992, pp. 26-38.

[6] Fortuin, C. M., Kasteleyn, P. W., and Ginibre, J., Correlation inequalities on some partially ordered sets, Communications in Mathematical Physics 22 (1971), 89-103.

[7] Jerrum, M., Two remarks concerning balanced matroids, submitted.

[8] Oxley, J. G., Matroid Theory, Oxford University Press, New York, 1992.

[9] Royle, G. and Sokal, A. D., The Brown-Colbourn conjecture on zeros of reliability polynomials is false, Journal of Combinatorial Theory, Series B 91 (2004), 345-360.

[10] Pemantle, R., Towards a theory of negative independence, Journal of Mathematical Physics 41 (2000), 1371-1390.

[11] Seymour, P. D. and Welsh, D. J. A., Combinatorial applications of an inequality from statistical mechanics, Mathematical Proceedings of the Cambridge Philosophical Society 77 (1975), 485-495.

[12] Sokal, A. D., The multivariate Tutte polynomial (alias Potts model) for graphs and matroids, in Surveys in Combinatorics 2005 (ed. Bridget S. Webb), Cambridge University Press, 2005, pp. 173-226.

[13] Wagner, D. G., Rank-three matroids are Rayleigh, The Electronic Journal of Combinatorics 12 (2005), \#N8 1-11.

[14] Wagner, D. G., Negatively correlated random variables and Mason's conjecture, arXiv:math.CO/0602648v1 (2006).

Department of Mathematics and Statistics, University of Canterbury, Christchurch, New Zealand

E-mail address: c.semple@math.canterbury.ac.nz

Merton College, University of Oxford, Oxford, United Kingdom

E-mail address: d.welsh@maths.ox.ac.uk 